\title{
Caracterización de las habilidades del razonamiento matemático en niños con TDAH
}

\section{Characterization of Mathematical Reasoning Skills in Children with ADHD}

\author{
Ángel Salvatierra Melgar* \\ Universidad César Vallejo, Lima, Perú \\ ORCID: https://orcid.org/0000-0003-2817-630X \\ Santiago Aquiles Gallarday Morales \\ Universidad César Vallejo, Lima, Perú \\ ORCID: https://orcid.org/0000-0002-0452-5862 \\ Yolvi Ocaña-Fernández \\ Universidad Nacional Mayor de San Marcos, Lima, Perú \\ ORCID: https://orcid.org/0000-0002-2566-6875 \\ Jessica Paola Palacios Garay de Rodríguez \\ Universidad Nacional Federico Villarreal, Lima, Perú \\ ORCID: http://orcid.org/0000-0002-2315-1683
}

Recibido 07-07-18 Revisado 12-08-18 Aprobado 15-12-18 En línea 04-01-19

\section{*Correspondencia}

Email: smelgara@ucv.edu.pe

\section{Citar como:}

Salvatierra, A., Gallarday, S., Ocaña-Fernández, Y., \& Palacios, J. (2019). Caracterización de las habilidades del razonamiento matemático en niños con TDAH. Propósitos y Representaciones, 7(1), 165-184. Doi: http://dx.doi.org/10.20511/pyr2019.v7n1.273

(C) Universidad San Ignacio de Loyola, Vicerrectorado de Investigación, 2019 


\section{Resumen}

El objetivo de la presente investigación fue caracterizar las habilidades del razonamiento matemático en niños con TDAH entre 7 a 9 años, el estudio inicia de la concepción de niños con necesidades especiales y se realiza una indagación antropológica en perspectivas del actor sobre las habilidades del razonamiento matemático y las entrevista a sus tres docentes acerca de sus dificultades, alteraciones y trastornos. El método de la investigación es el trabajo de campo etnográfico. Los resultados muestran carencia de habilidades matemáticas específicamente en cálculo numérico; apreciándose desorden, combinación de operación simultáneamente entre adición y sustracción, déficit de atención durante la lectura de un problema por lo que no logran sistematizar, identificar los datos para las operaciones algorítmica

Palabras clave: Habilidades, razonamiento matemático, niños con TDAH.

\section{Summary}

The objective of the present research was to characterize the mathematical reasoning skills in children with ADHD between 7 and 9 years old. The study begins with the conception of children with special needs and an anthropological research is carried out in perspectives of the actor on mathematical reasoning skills and interviews of their three teachers about their difficulties, alterations and disorders. The research method is an ethnographic fieldwork. The results show lack of mathematical skills specifically in numerical calculation; observing disorder, combination of simultaneous operation between addition and subtraction, attention deficit during the reading of a problem so they fail to systematize, identify data for algorithmic operations

Keywords: Skills; Mathematical Reasoning; Children with ADHD.

\section{Introducción}

La habilidad posee diversas definiciones dependiendo de los lentes desde el cual se quiere orientar, en cuanto a: la solución de problemas, en el trabajo, en la comunicación, en el uso de la tecnología, en el deporte, en el comportamiento social, en la participación política, en psicosociales, etc. Por tanto, la habilidad constituye un constructo social donde lo teórico es transferido a la práctica como refiere Rigby y Sanchis (2006), desde esta postura, la habilidad se identifica como los conocimientos técnicos o prácticos, implicando la aplicación del conocimiento teórico a situaciones concretas dentro de un contexto.

Recurriendo a la postura de habilidades por parte de la Organización Mundial de la Salud (OMS) quien enmarca dentro de un plano humano; como a la capacidad del comportamiento positivo de las personas y capaz de adaptarse al comportamiento de los demás y ser parte de los retos cotidianos WHO (1997), por tanto, el camino para una buena educación basada en habilidades propicia una nueva forma y cultura de aprendizaje, cuyo norte está orientada la preparación para la vida UNESCO (2004). Bajo esta línea, Portillo (2017) manifestó, "Cada estudiante progresa a diferentes ritmos en el dominio de una habilidad implica saber atender oportunamente sus necesidades y la personalización de las trayectorias escolares" (p.10), he allí el papel de los actores de la educación, donde cada estudiante es un ser individual con procesos de aprendizaje variados, de ser necesario adoptar cambios en la práctica docente que responda a las necesidades múltiples del estudiante, repercutiendo a una reorganización educativa.

\section{Habilidades y el razonamiento matemático.}

El razonamiento matemático, conlleva a un razonamiento formal de manera consciente permitiendo la solución de problemas y generar las conclusiones pertinentes, al respecto Chevallard, Bosch y Gascón (1997) refiere que las matemáticas, se encuentran presentes en 
nuestra vida cotidiana, en el quehacer diario por medio de los objetos técnicos, en compras diarias, pagos de servicios, juegos en casinos, en repartos de algún producto en la mesa, en cálculos próximos de longitud, peso de algún objeto, otros. El conocimiento matemático es una de las disciplinas que permite fortalecer la capacidad de razonamiento, en cuanto a; abstracción, toma de decisiones, análisis, síntesis, predecir, sistematizar y resolver problemas de orden lógico o heurístico, que permite una formación básica a nivel cultural para el desenvolvimiento cotidiano. Asi mismo, Defaz (2017) manifiesta que gracias al pensamiento, la observación y la intuición que conlleva a la representación mental y la imaginación del razonamiento lógico para establecer relaciones de deducción, aplicar el significado a situaciones simbólicas e inferir, también permite el desarrollo de procesos didácticos como: la inducción a partir de datos o casos concretos, la deducción bajo el desgarro de lo general a los procesos específicos; estos argumentos son claves para el pensamiento Heurístico.

Dentro del plano académico, las habilidades del razonamiento matemático es entendida a la forma de utilizar los números a situaciones con sentido práctico durante el desarrollo de las operaciones básicas, haciendo uso de símbolos, interpretando y resolviendo problemas relacionadas a la vida cotidiana y laboral Calzada (2014), asimismo, la expresión del razonamiento, está ligado a la vocalización de símbolos, a la transposición del mundo abstracto a los enunciados verbales para su comunicación según al propósito y la naturaleza de situaciones. Sin embargo, la interpretación, está basada en la comprensión del formato grafo, el significado de la información simbólica y numérica conlleva a la resolución de problemas quien se traduce a situaciones reales y concretas orientando la comprensión e interpretación en la selección de estrategias y procedimientos adecuados orientados bajo la secuencia heurística durante el afrontamiento.

\section{Niños con necesidades educativas especiales.}

En el sistema educativo peruano, las instituciones están representadas tanto en las entidades privadas como públicas por un conjunto de personas con roles específicos, por su parte MineduPerú (2018) refiere que la escuela es un lugar de oportunidades de aprendizaje y participación en torno al niño, independientemente de sus condiciones personales, sociales o culturales, ellos son valorados en sus diferencias personales. Es labor del docente lograr que los niños se sientan parte del grupo, interactuando profesionalmente con los niños a través: la orientación, el seguimiento, guiando y evaluando el proceso de aprendizaje. Al respecto Fernández y Sarramona (1985) manifiestan que el docente en su ejercicio profesional, suele compartir experiencias de aprendizaje con niños que muestran o se encuentran en inicio de algún tipo de problemas de aprendizajes dificultando su normal desarrollo académico y convivencia social. Al respecto Moriña y Almudena (2017) refirieron que muchos de los docentes no se encuentran suficientemente formados para responder a las necesidades de estudiantes con algunas necesidades especiales, siendo necesario recibir alguna especialización o insertar en el currículo de formación superior. Por su parte Warnock (1987), identificó cuatro grandes necesidades del docente durante su práctica pedagógica: (a) niños con problema de audición, visión o movilidad, (b) desventajas educativas, (c) dificultad de aprendizaje y (d) dificultades emocionales y conductuales.

Debido a las múltiples y variedades necesidades especiales educativas en nuestro contexto el INEI (2014) detectó una recurrencia frecuente de casos de Trastorno por Déficit de Atención e Hiperactividad (TDAH) o desorden de atención en niños. De acuerdo a Fernández, Arjona, Arjona y Cisneros (2011) un promedio de 5\% de los niños del mundo presentan este trastorno, que se manifiesta con mayor frecuencia en niños que niñas, de cada tres niños hay una niña; así mismo Fernádez (2011) manifiesta que el TDAH, se presentan antes de los 7 años y se mantiene en el tiempo generando deterioro en el rendimiento académico y déficit en el desarrollo de habilidades sociales, actividades cotidianas y emocionales.

Los niños con déficit de atención e hiperactividad, según Portela, Carbonell, Hechavarría 
y Jacas (2016), se deben en primer lugar a factores hereditarios, representado por el $80 \%$ de los casos; biológicos adquiridos durante los periodos prenatal, perinatal y posnatal; neurofisiológico, activaciones cerebrales, reducción del metabolismo, activación de flujos sanguíneos y actividad eléctrica en la corteza sensorio-motora. En segundo lugar, factores genéticos, observado en tasas de concordancia para mellizos (gemelos dicigóticos) e idénticos (gemelos monocigótico), psicosociales y ambientales; conforman la psicopatología de los padres, estrés psicosocial de la familia, mala alimentación, alcoholismo y video juego. En tercer lugar, el factor neuroquímico, generado por flujo cerebral por tomografía de fotón, déficit de regulación de neurotransmisores como la dopamina y norepinefrina en la corteza frontal, la serotonina en el control de los impulsos. Como se puede tomar atención, son niños que requieren de tratamientos especializados debido a sus múltiples causas; sin embargo, el presente estudio, está orientado a describir y caracterizar la forma de aprendizaje de los niños que presentan TDAH, esta caracterización está orientado por lo que necesitan más tiempo y mantenerlos en control para que puedan terminar su tarea.

\section{Habilidades y Razonamiento Matemático en Niños NEE.}

Los componentes identificados en las habilidades del razonamiento matemático de acuerdo a las Bases Curriculares de la Matemática Funcional para Estudiantes que presentan (NEE) de Chile y desarrollados por el Ministerio de Educación (2016) quien organizó y caracterizo las necesidades de los niños en cuatro categorías específicas: Resolver problemas, Argumentar y comunicar, Modelar y Representar; estos componentes curriculares, se encuentran diseñados específicamente para los niños con NEE, expuestos en organizadores con actividades prácticas orientadas al desarrollo y promoción de sus habilidades innatas. Los niños con TDAH muestran deficiencias en adquisición de habilidades lingüísticas y matemáticas frente a los niños normales, puesto que no consiguen obtener su rendimiento de acuerdo a su edad y su coeficiente intelectual Zentall (2007), debido al déficit de sus funciones ejecutivas (FE) asociadas a la búsqueda organizada, la flexibilidad de acciones y el pensamiento por ende al razonamiento lógico. Según Gratch (2009) las situaciones específicas como: numeración y cálculo, muestran la falta de exactitud o de precisión durante los cálculos aritméticos por la confusión de signos. En cuanto a la resolución de problemas; no logran sistematizar los datos implícitos y presentar la secuencia para la solución del problema, presentan carencia en el ordenamiento de dígitos, lectura, lenguaje matemático y secuencias de los procesos mentales para la sentencia del problema, todos y otros aspectos se explica por la carencia de estrategias maduras de conteo, generando conglomeraciones o embotellamiento en la memoria de trabajo debido a la carencia de atención y concentración que induce a consecuencias graves en el aprendizaje de la matemática Barkley (1997), ademas presentando resultados o logros deficientes no alentadores tanto para el docente como para sus padres.

Con aras de mantener o centrar la atención de los niños con TDAH, se recomienda manipular materiales educativos concretos con gran cantidad de estímulos o textos con sentencias claras, orientadoras, evitando la distracción y generar estímulos significativos Miranda, Alba y Taverner (2009), puesto que principal característica de estos niños es la perdida de atención y concentración a las tareas encomendadas, se manifiestan dificultades en la solución de problemas, pese a que leen muy bien en su mayoría no entienden ni identifican los datos implícitos; no logran identificar a las operaciones aritméticas induciendo a una confusión entre los operadores, generando tres tipos de conocimiento: el declarativo de conceptos, consta de operaciones y estrategias de resolución. El procedimental, comprende de la aplicación del conocimiento declarativo y la coordinación de procesos cognitivos y el conocimiento condicional; que comprende en la selección de estrategias más adecuadas.

\section{Método}

Para el desarrollo del estudio, se procedió por la metodología cualitativa etnográfica permitiendo conocer el comportamiento directo de los sujetos Hernández, Fernández, y Baptista (2014). Se identificó a los niños con TDAH utilizando la lista de chequeo del DSM_IV, quienes fueron 
sujetos de observación, luego se realizaron las entrevistas semiestructuradas a 3 docentes con quienes empalmamos la interacción individualizada bajo códigos de identificación, previo los consentimientos informados Izcara (2014), así mismo los docentes de aula comunicaron sobre los comportamiento, vivencias, experiencias de los niños con TDAH en el aula así como las capacitaciones en educación inclusiva a los que han participado. Se entrevistó a un especialista. Se utilizó la técnica de observación para registrar las conductas de los niños, comportamientos como al salir del aula se percataban de la presencia del investigador, situación no planificada, y que se presentaron, por la curiosidad innata de los niños Aragón, Natalia, Marcia, y Juan (2016) ante ello, de manera voluntaria al acercarse se les realizó preguntas; ¿Cuál es la serie de su agrado, el último juego virtual, quienes son los niños: atentos, estudiosos, juguetones y deportistas. Estos datos fueron tomados de manera cuidadosa y organizada mediante códigos; que contribuyeron a identificar a las subcategorías y categorías, para el proceso del análisis e interpretación de los datos, se procedió a utilizar la técnica de la triangulación múltiple Aguilar y Barroso (2015), a su vez fueron organizados en unidades hermenéuticas expuestas al programa del Atlas.ti obteniendo las redes semánticas.

\section{Procedimiento}

Identificados los entes de estudio, se procedió a la recolección de los datos de manera paulatina previo acuerdo con las docentes. La participación dentro del aula fue de forma sutil en tres momentos sin participación alguna durante las sesiones de aprendizaje; puesto que se deseaba identificar las acciones más naturales, puntuales y específicas acerca de las conductas de los niños puestos a observación. El rol del investigador fue eminentemente observador durante la explicación de las docentes en el desarrollo de las actividades de clases trabajadas con los niños, en la conformación de los grupos de estudiantes para sus tareas específicas, los comportamientos entre los miembros de su grupo y entre los otros miembros, las acciones de aprendizaje en el área de matemática, participación para la sentencia solicitada por la maestra, antes de la finalización de su actividad y después de la finalización.

Solo al final de cada sesión se registraron las conductas observadas en el cuaderno de campo, asimismo se procedió a las entrevistas semiestructurada a las docentes de aula en un tiempo de 10 a 15 minutos, quienes mostraron preocupación por: la carencia de atención y concentración, demasiada hiperactividad, distracción con facilidad, carencia de habilidades sociales, uso de materiales diferenciados.

Finalmente, se concluye el trabajo de campo con la entrevista al especialista en conductas especiales en niños; dichos aportes fueron sistematizados y organizados por la técnica de la triangulación, detectándose dos aspectos categóricos con sus respectivos componentes: a) Niños con necesidades educativas especiales TDAH, con componentes: a.1) procesos cognitivo, a.2) Atención, a.3) Concentración, a.4) Aprendizaje y a.5) Pensamiento lógico. b) Habilidades y razonamiento matemáticas con niños TDAH, con componentes: b.1) Operaciones aritméticas, b.2) Resolución de problemas, b.3) Medios y materiales, b.4) Materiales concretos y b.5) Hojas de trabajo con diseños especiales.

\section{Resultados}

El procesamiento de los datos reportados por el programa del Atlas.ti_V_7.5.4 a partir de la sistematización de las entrevistas de las docentes y especialista, datos del cuaderno de campo del investigador y las aproximaciones teóricas señaladas en la introducción del estudio, muestran los principales nodos en las redes semánticas en función de las categorías y las conexiones de los fragmentos definidos por colores, para luego identificar las conexiones orientadoras con respecto a los resultados de las características y necesidades, así mismo de las características cognitivas del niños con TDAH en el aprendizaje de la matemática. 


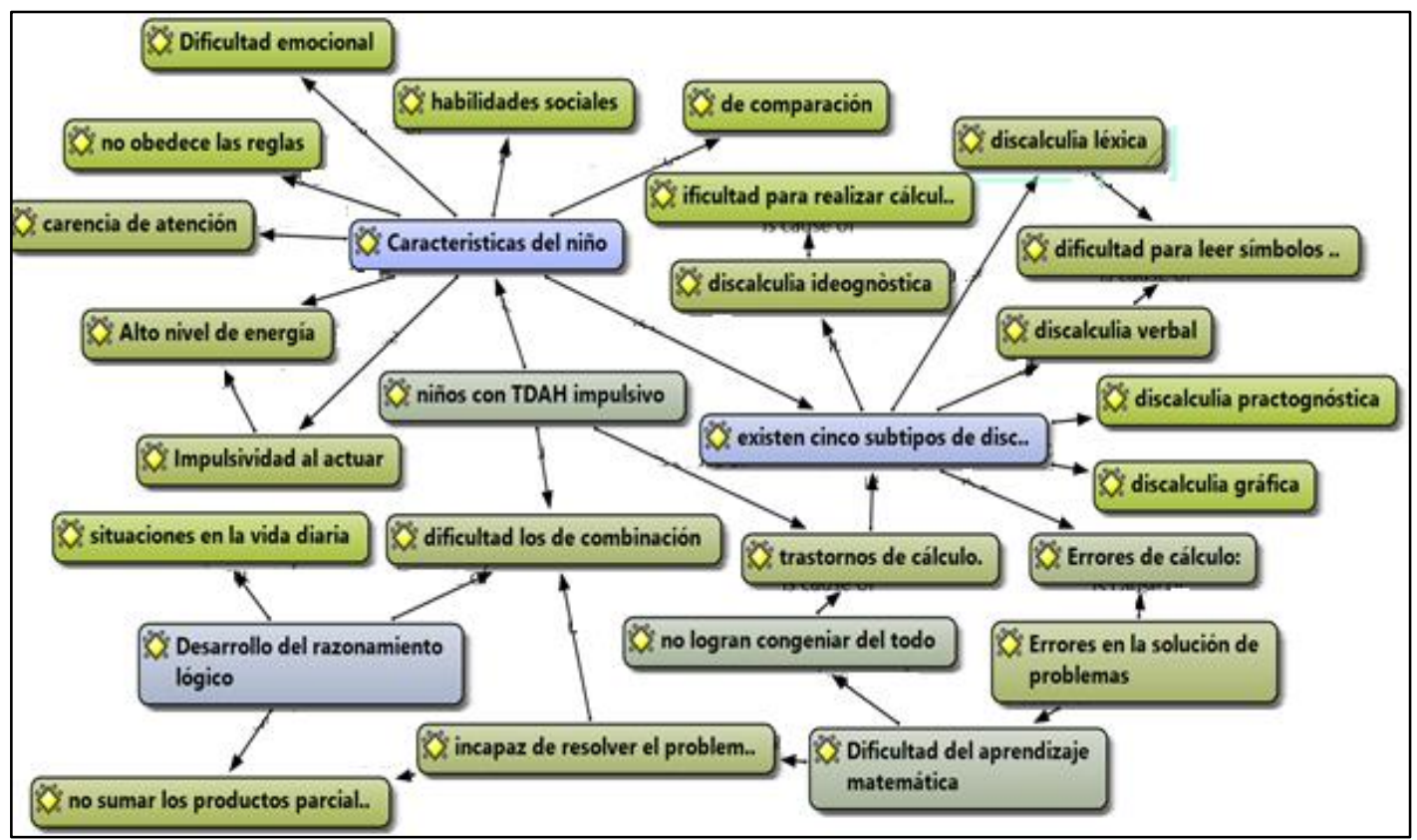

Figura 1. Características y necesidades de los niños con TDAH en el proceso de aprendizaje de matemática.

En la red semántica expuesta en la figura 1, se muestra el nodo centralizado en las características del niño con trastorno de TDAH, identificadas por la postura de las docentes asociadas por la observación del investigador, una de las características que llamo la atención a pesar que carecen de habilidades sociales, puesto que no son aceptados al grupo por su comportamiento Fernández (2011), es que los niños durante la hora de recreo y juegos libres, demuestran solidaridad, colaboración, participación, hasta incluso son capaces de compartir sus loncheras a quien lo solicita, lo que no ocurre dentro de las aulas; donde sus compañeros los rechazan debido a sus comportamientos como emitir chillidos, transitar de un lugar a otro, simular con sonido y movimientos de sus manos al recordar un juego.

Durante los acuerdos impartidos para el trabajo en equipo por parte de la docente, los niños fijan su atención un promedio de 5 a 7 segundos y su primera reacción es mirar a su compañero de al costado y llamar la atención con algún objeto que muestra en el tablero, perdiendo la emisión de las reglas de la docente (Portela, Carbonell, Hechavarría, \& Jacas, 2016), generando incomodidad entre los integrantes de su grupo que permanentemente, se distraen con facilidad mostrando un alto nivel energético a pesar que sus compañeros tratan de regular sus conductas persiste en su acción desprendida. Con respecto al aprendizaje en el área de matemática se ha detectado que son muy hábiles en acciones comparativas con materiales concretos; sin embargo, muestran dificultad en las estructuras de cambio; al remplazar un objeto por una decena, posicionar cantidades y formar otras estructuras numéricas. En el proceso de la triangulación se han detectado la carencia de algunas habilidades aritméticas en las operaciones elementales de cálculo como las presentes en la discalculia (Martínez, Calzadilla, \& Cruz, 2017) lo cual implica la imposibilidad para comprender el significado de los números y las cantidades, generando errores comunes en la adquisición de habilidades básicas de adición, sustracción, multiplicación y división, el estudio nos muestra que los errores más frecuentes cometidos por los niños en las operaciones básicas de la adición y sustracción son asociados a la discalculia ideognóstica (García, Santana, Soria \& Vila, 2016), frecuente, no consideran el valor que llevan para la siguiente operación, olvidan el valor prestado (canjeado) de la cifra del minuendo posterior, asimismo se detectó la presencia de discalculia léxica (Martínez, Calzadilla, \& Cruz, 2017) quienes mostraron dificultad o confusión para leer e identificar los símbolos de operadores matemáticos en operaciones combinadas, repercutiendo en el nivel de comprensión a los problemas verbales; sin embargo, con respecto a la discalculia practognósica, no se observó 
demasiada variación entre sus compañeros, más aun fueron los que mostraron dominio en la manipulación de objetos en la comparación de tamaño y forma mencionando las características entre ellos. Para el aprendizaje de los niños con TDAH, se recomienda la manipulación de materiales concretos, se debe seleccionar las estrategias más adecuadas para fijar su atención (Miranda, Alba, \& Taverner, 2009).

Bajo estos argumentos, los niños identificados con TDAH, presentan dificultades durante su aprendizaje de las matemáticas, en los casos de problemas de matemática literales, el hecho que no logran congeniar o terminar y entender al enunciado del problema debido a la distracción y desatención se muestran incapaces de plantear, afrontar y resolver el problema, por lo que, durante el proceso de resolución, no logran combinar los datos y los enunciados implícitos del problema. Bravo y Urquizo (2016) el razonamiento lógico es la competencia de usar los números eficazmente, analizar problemas lógicamente e investigar soluciones; bajo esta postura, el niño con TDAH muestra limitaciones en su razonamiento lógico, que lo llevarian a tener problemas para enfrentar situaciones lógicas de la vida diaria, teniendo en cuenta que las matemáticas se enmarcan en el mundo cultural, natural y social en la que las personas viven; por tanto, cabe la necesidad de asumir transversalmente la alfabetización matemática (MINEDU, 2015).

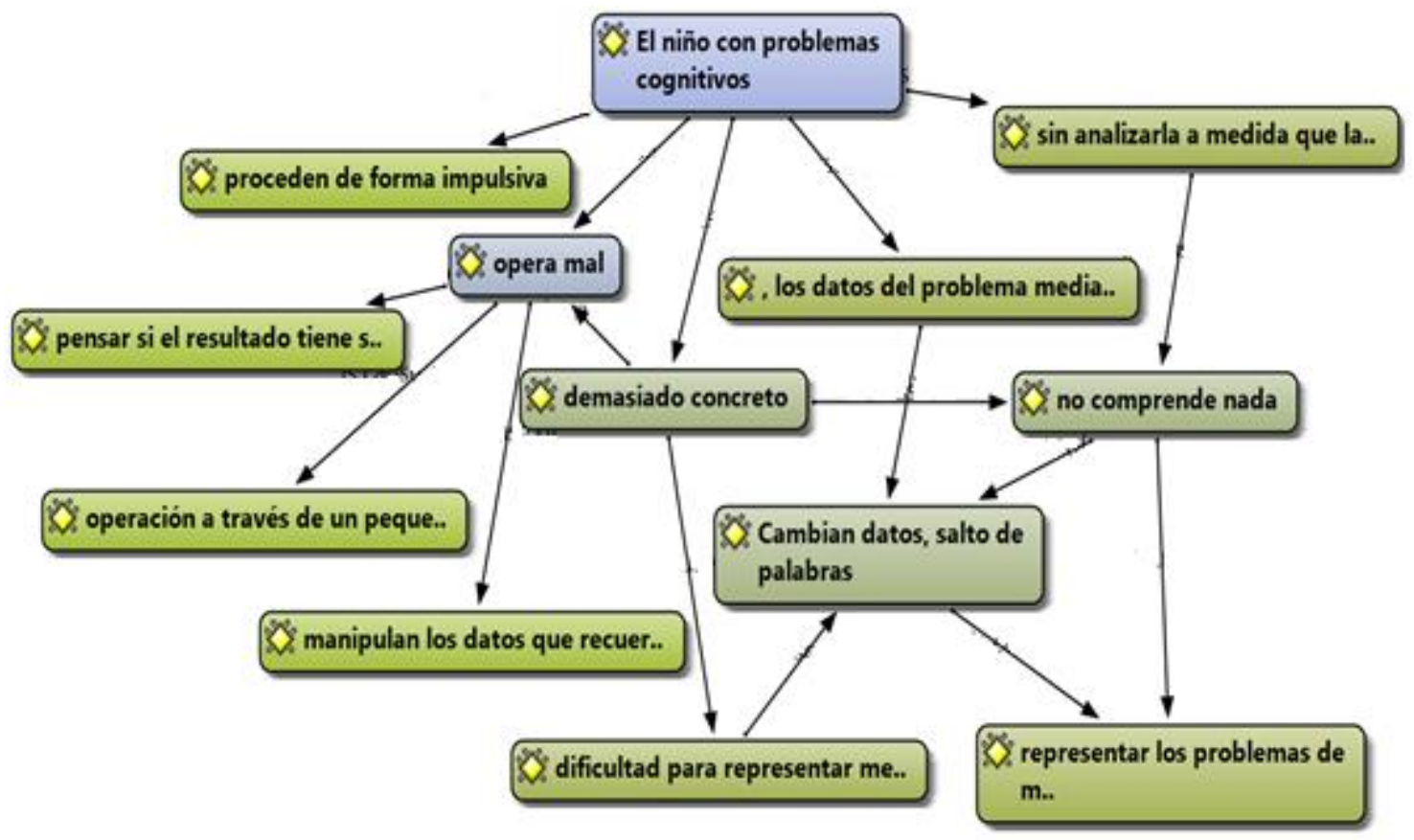

Figura 2. Caracterización cognitiva de niños con TDAH en el aprendizaje de la matemática.

Con respecto a los problemas cognitivos en los niños con TDAH, se manifiestan los trastornos de neurodesarrollo teniendo lugar al déficit de atención durante el proceso de aprendizaje Alvárez y Pinel (2015); se ha observado que el niño con trastorno de atención al iniciar una actividad inician otras actividades dejando de lado su actividad previa y así otra actividad, no terminan una actividad sin antes la presión o exigencia de la docente, o en todo caso realizan sus operaciones de manera herrada; puesto que en las operación aritmética se equivocan al realizar la adición confunden con los procedimiento de la sustracción o combinan ambas operaciones. Para Granados, Figueroa y Valásquez (2016) la atención es uno de los procesos cognitivos y conductual, que consiste en la fijación y concentración selectiva hacia un objeto el cual determinara la calidad de respuesta, bajo esta postura el niño con TDAH carece de atención durante el aprendizaje de las matemáticas sin antes de terminar con la sentencia señalada inicia de manera exploratoria otra actividad de manera impulsiva, esta acción hace que el niño se equivoque y terminada presentando resultados de manera ligera sin antes de pensar a veces certero; como es el caso que restan de un número menor a un mayor, asimismo no logran organizar 
el texto de un problema, por lo que no logran descubrir un algoritmo y resolverlos bajo ciertas secuencias de acuerdo a las operaciones de adición; llevando y sustracción; con canje, asimismo en problemas de cambio y transposición de objetos.

Estos niños, por sus características demuestran ser muy concretos, necesitan algún material de aprendizaje para su manipulación, o fichas guiadas con datos puntuales coloridos para activas su atención mediante dibujos, guiadores, secuencias de procedimientos para alcanzar el fin; como es el caso de cuadrados y triángulos mágicos, sudokus, desplazamiento de figuras, entre otros. A pesar que el niño lee correctamente las situaciones problemáticas, aparentemente no entiende lo que lee, al pedirle la sistematización de los datos implícitos del problema ellos cambian constantemente por otros o una sentencia por otra, no distinguen el tipo de operación aritmética a realizar; detectado el operador lo pintan y lo repintan de colores o repasan remarcando tratando de entender al problema, se debe por la incapacidad de representar mentalmente, pero sin embargo a leerlos el mismo problema de manera pausada y contextualizada ellos demuestran una rapidez y habilidad para su interpretación y solución del problema e incluso relacionan con la vida real generando algunas referencias o acontecimientos. Al respecto Ríos \& López (2017) manifiesta que el trastorno es una dificultad en la habilidad de la lectura, escritura y cálculos aritméticos y que su manifestación variada y diferenciada en los niños es principalmente en lo académico.

\section{Discusión}

El estudio, muestra el análisis a uno de los componentes de las necesidades educativas especiales en niños con Trastornos por Déficit de Atención e Hiperactividad del TDAH, quienes se hacen presente entre 2 a 3 niños en promedio por aula, tal como manifiesta el INEI (2014) y Fernández, Arjona, Arjona, y Cisneros (2011) que un promedio del 5\% de niños presentan el mencionado trastorno que dificulta el aprendizaje por la carencia de atención, de acuerdo a Portela, Carbonell, Hechavarría y Jacas (2016) puesto que se debe a múltiples factores como: hereditarios, biológicos o en el proceso del pre o post parto; ya que en los primeros años de vida en niño se desenvuelve de manera normal, a medida que la niños se le asigna consignas muestra cierto agotamiento, en el caso de tareas, operaciones aritméticas, actividades de trazo, cortes; al mismo tiempo no muestra la atención fijada a una actividad, a medida que las consignas se muestran de manera lógica, aproximadamente entre los 6 a 7 años, el niño con TDAH las acciones cognitivas se reflejan de manera selectiva frente a sus demás compañeros; como es el caso, de mirar a sus alrededores cuando la maestra está explicando, no termina de copiar lo que está escrito en la pizarra, coloreados desalineados e inconcluso, por su parte Zentall (2007), menciona que se debe al déficit de sus funciones ejecutivas, repercutiendo a situaciones específicas como: numeración.

Según Gratch (2009), el niño muestra confusiones en el momento de realizar las operaciones de adición y sustracción al no poder identificar el tipo de operación aritmética, repercutiendo en sus demás niveles educativos por ende en su desenvolvimiento académico. Frente a esta necesidad que es latente, observable, desde la maestra hasta las entidades como es el caso de la Organización Mundial de la Salud (OMS), Ministerios y Organizaciones, se debe priorizar en la inversión y prestar el servicio profesional especializado a los niños con estos tipos $\mathrm{y}$ otros trastornos que posiblemente sean los problemas que aqueja en el bajo rendimiento académico, corroboramos lo manifestado el estudio de Moriña y Almudena (2017) los docentes no se encuentran suficientemente formados y preparados para responder a estas necesidades. En cuanto al aprendizaje de las áreas específicamente en el área de matemática, los niños con las características descritas, requieren de gran apoyo para lograr las capacidad y competencias, por su particularidad estos niños fácilmente pierden la concentración ya que permanentemente se distraen y confunden o se olvidan con facilidad al momento de realizar las operaciones o consignas, al respecto Gratch (2009) manifiesta que es preocupante ver como los niños con trastorno muestran dificultades en: numeración y cálculo; se observó de manera incito ya que frecuentemente el niño solicita apoyo a sus compañeros pero les cuesta entender tanto en la explicación por sus compañeros durante el trabajo grupal y por la maestra, no logran integrar los 
enunciado o situación matemáticas, a identificar los datos y el tipo de operaciones a realizar. Frente a esta situación, es necesario la asistencia profesional, dotando de materiales diseñados; como: cuadernos de trabajo, videos educativos, entre otros, el cual corroboramos con los hallazgos de Ríos y López (2017) los niños con trastorno, es una dificultad en la habilidad de la lectura, escritura y cálculos aritméticos e implementar el área psicopedagógica para atender múltiples necesidades de aprendizaje y conductas en cada Institución Educativa como lo hacen otros países.

\section{Referencias}

Aguilar, S., \& Barroso, J. (2015). La triangulación de datos como estrategia en investigación educativa. Pixel-Bit. Revista de Medios y Educación, 17(47), 73-88. Doi: https://doi.org/10.12795/pixelbit.2015.i47.05

Alvárez, S., \& Pinel, A. (2015). Transtorno por déficit de atención con hiperactividad en mi aula de infancia. Reop. Revista Española de Orientación y Psicopedagogía, 26(3), 141-152 Doi: https://doi.org/10.5944/reop.vol.26.num.3.2015.16406

Aragón, L., Natalia, J., Marcia, E., \& Juan, V. (2016). Acercar la ciencia a la etapa de infantil: experiencias educativas en torno a talleres desde el Grado de Maestro en Educación infantil. Revista Iberoamericana de Educación, 72, 105-128. Recuperado de: http://hdl.handle.net/10498/20057

Barkley, R. (1997). ADHD and the nature of self-control. New York: Guilford Press.

Bravo, P., \& Urquizo, M. (2016). Razonamiento lógico abstracto e inteligencia emocional: trayectorias en la formación de estudiantes universitarios. Sophia, Colección de Filosofía de la Educación, 21, 179-208. Doi: https://doi.org/10.17163/soph.n21.2016.08

Calzada, A. (2014). Guía de Evaluación de la competencia básica en razonamiento matemático. Sevilla: Junta de Andalucía

Chevallard, Y., Bosch, M., \& Gascón, J. (1997). Yves Chevallard et al. Estudiar matemáticas. El eslabón perdido entre enseñanza y aprendizaje. Cuadernos de educación. Para profesores, padres y alumnos (vol. 22). España: Horsori.

Defaz, G. (2017). El desarrollo de habilidades cognitivas mediante la resolución de problemas matemáticos. Journal of Science and Research, 2(5), 14-17. Doi: https://doi.org/10.26910/issn.2528-8083vol2iss5.2017pp14-17

Fernádez, C. (2011). Determinación de las necesidades educativas especiales. Mexico D.F: Trillas.

Fernández, A., \& Sarramona, J. (1985). La educación Constantes y problémicas actuales (12 ed.). Barcelona: CEAC.

Fernández, A., Arjona, P., Arjona, T., \& Cisneros, A. (2011). Determinación de las Necesidades Educativas Especiales. Mexico: Trillas.

García, N., Santana, A., Soria, B. H., \& Vila, M. (2016). Neuropsicología y bases neuronales de la discalculia. Tercer Congreso virtual de Ciencias Morfológicas. Cuba. Recuperado de: http://www.morfovirtual2016.sld.cu/index.php/Morfovirtual/2016/paper/viewFile/110/1 47

Granados, D., Figueroa, S., \& Valásquez, A. (2016). Dificultad de atención y competencias de investigación en estudiantes universitarios de psicología. CNEIP. Enseñanza $e$ investigación en psicología, 21(2), 11. Recuperado de: http://www.redalyc.org/articulo.oa?id=29248181003

Gratch, L. (2009). El trastorno por déficit de atención (ADD_ADHT). Clínica, diagnóstico y tratamiento en la infancia, la adolescencia, y la adultez. Buenos Aires: Médica Panamericana.

Hernández, R., Fernández, C., \& Baptista, M. (2014). Metodología de la Investigación (6 ed.). México: McgrawHill.

INEI. (2014). Primera encuesta nacional especializada sobre discapacidad 2012. Lima: INEI

Izcara, S. (2014). Manual de Investigación Cualitativa (1 ed.). Mexico: Fontamara.

Martínez, M., Calzadilla, O., \& Cruz, M. (2017). La discalculia: Un reto para la enseñanza de la matemática. En Memorias Compumat 2017, 127. La Habana: Cujae. 
Ministerio de Educación. (2015). Alfabetización en Matemáticas y Ciencia. En E. P. Resultados. Perú. Recuperado de: http://repositorio.minedu.gob.pe/bitstream/handle/123456789/44/024.\%20Una\%20apro ximaci\%C3\%B3n\%20a\%20la\%20alfabetizaci\%C3\%B3n\%20matem\%C3\%A1tica\%20y $\% 20$ cient $\%$ C3\%ADfica\%20de $\% 201$ os $\% 20$ estudiantes $\% 20$ peruanos $\% 20 \mathrm{de} \% 2015 \% 20 \mathrm{a}$ $\% \mathrm{C} 3 \% \mathrm{~B} 1$ os \%20Resultados\%20del\%20Per\%C3\%BA\%20en\%201a\%20evaluaci\%C3\% $\mathrm{B} 3 \mathrm{n} \% 20$ internacional $\% 20 \mathrm{PISA}$.pdf? sequence $=1$

Ministerio de Educación. (2018). Educación Inclusiva. Perú. Recuperado de: http://www.minedu.gob.pe/educacioninclusiva/

Ministerio de Educación. (2016). Bases Curriculares. Matemática Funcional para Estudiantes que presentan Necesidades Educativas Especiales. Chile: Reforma Educacional. Recuperado de: http://especial.mineduc.cl/wp-content/uploads/sites/31/2016/10/Manual_Docente_Matematica-2016-1.pdf

Miranda, A., Alba, M., \& Taverner, M. (2009). Habilidades matemáticas y funcionamiento ejecutivo de niños con trastorno por déficit de atención con hiperactividad y dificultades del aprendizaje de las matemáticas. Psicothema, 21(1), 63-69. Recuperado de: http://www.psicothema.es/pdf/3596.pdf

Moriña, A., \& Almudena, C. (2017). Educación Inclusiva y Enseñanza Superior desde la mirada de estudiantes con Diversidad Funcional. RIDU Revista Digital de Investigación en Docencia Universitaria, 11(1), 20-37. Doi: http://dx.doi.org/10.19083/ridu.11.528

Portela, A., Carbonell, M., Hechavarría, M., \& Jacas, C. (2016). Trastorno por déficit de atención e hiperactividad: algunas consideraciones sobre su etiopatogenia y tratamiento. Revista Médica de Santiago de Cuba, 20(4). Recuperado de: http://www.medisan.sld.cu/index.php/san/article/view/813

Portillo, M. (2017). Educación por habilidades: Perspectivas y retos para el sistema educativo. Educación, 41(2). Doi: http://dx.doi.org/10.15517/revedu.v41i2.21719

Rigby, M., \& Sanchis, E. (2006). The concept of skills and its social construction. European Journal of Vocational Training, 37(1), 22-33. Recuperado de: http://aei.pitt.edu/44901/1/37-2006.pdf\#page $=24$

Ríos J. A., \& López C. R. (2017). Neurobiología de los trastornos del aprendizaje y sus implicaciones en el desarrollo infantil: propuesta de una nueva perspectiva conceptual. Psicoespacios, 11(19), 174-192. Doi: https://doi.org/10.25057/21452776.942

UNESCO. (2004). Grupo Inter-Agencias sobre habilidades para la vida en EFA. Paris: UNESCO

Warnock, M. (1987). Special Educational Needs. Report of the Committee of Enquiry into the Education ofHandicapped Children and Young People. London: HMSO.

WHO. (1997). Conclusions from a United Nations Inter-Agency Meeting. Geneva: World Health Organization

Zentall, S. S. (2007). Math performance of students with ADHD: Cognitive and behavioral contributors and interventions. In D. B. Berch \& M. M. M. Mazzocco (Eds.), Why is math so hard for some children? The nature and origins of mathematical learning difficulties and disabilities (pp. 219-243). Baltimore, MD, US: Paul H Brookes Publishing. 